\title{
Exploring the grounding problem of OPGW in substation of Longmen framework
}

\author{
Zhu Wei, Duan Canyi, Zhang Fengfeng, Lin Dinghao \\ Email address:Sanmenxia Power Design Co., Ltd., Sanmenxia City, Henan Province, China \\ lyw1301@163.com
}

\begin{abstract}
Keywords: OPGW,Substation,Grounding,Research.
Abstract. OPGW with functions of lighting proof and communication is widely used in the construction of power network. For optical fiber composite overhead ground wire (OPGW) [1], it is required to achieve the separation of wire and signal after the introduction of the substation structure; at the same time, the grounding for lightning proof is also required because of the frequent occurrence of the burnout of the optical cable and short circuit due to the electric discharge and lighting as a result of the problems in the grounding device or construction [2]. This paper analysis some common advantages and disadvantages of OPGW structure grounding methods.In combination with the practical conditions of the project, the solution "Enhanced the grounding method" is brought up and solve the practical problem.
\end{abstract}

\section{Introduction}

OPGW （Optical Fiber Composite Overhead Ground Wire ） is transmission line earth wire,which is the main telecommunication transmission line of power dispatching. production management,replay protection, automatic dispatching information.Compared with another overhead grounding wire of transmission line and OPGW vulnerable to lightning strikes [3] .

As OPGW cable is different from ordinary cable, OPGW is required to achieve the separation of wire and signal after the accessing of the substation structure, introducing downwards to the structure, and connect with non-mental entrance cable in the connection box, Then enter the substation communication device.Because the problem of structures grounding device or construction problems, causing ground gap discharge occurs when lightning or circuit fault, although adopted a variety of measures, but burnt cable accidents still occur.

\section{The principle of OPGW into substation}

When designing the grounding method of OPGW to the substation structure, the consideration shall be given to four major principles.

1) It is required to guarantee the current shall flow through OPGW into the ground smoothly in case of the short circuit or lightning strike without the burnout of OPGW [4].

2) Unclean connection point between the appearance of OPGW with all the mental parts of the structure, to avoid the hidden intermittent discharge danger of between the appearance of OPGW with all the mental parts of the structure $[5]$.

3 ) When measuring the grounding resistance at the substation on a regular basis, it is easy to disassemble the ground wire from the ground grid of the substation.

4) The lead-in of OPGW cable can be installed without any damage on substation equipment.

The common earth wire and OPGW has the same function on lighting protection, OPGW cable lead to substation grounding power net meet compliance with related regulation,normally damage on substation equipment won't be occur. 


\section{Analysis of grounding and protection of OPGW}

After inquiries and investigations, grounding and protection of OPGW should be given in 5 ways, below is details description and analysis.

1) OPGW is connected directly to the steel pole on the top of the structure or grounding device. Such grounding is often used under the situations of the insufficient area of connection or for the problem in the grounding construction. In case of the grounded short circuit or the lightning strike happened to the system, due to the less resistance of the ground grid of the substation (Not higher than $5 \Omega$ ) or the longer tension resistant section of OPGW, the maximum impulse current appears at the connection to the substation structure, resulting in the high temperature at the connection, so that the outer aluminum alloy fuse of OPGW is burned out $[6]$. The method adopted for such connection is the same as that for the ordinary ground wire, and also is the earliest method for introduction of OPGW into the substation and seldom used separately nowadays. There is the improvement method by increasing the contact area of the ground device, but still have hidden danger.

2) OPGW is introduced from the top of the structure to the cable rack with the suspended or insulated padding plate, and connected to the grounding device with the drainage wire. By such method, when introducing the OPGW downwards from the top of the structure, the exterior of OPGW often makes the irregular contact with the metal member of the structure, and the stronger induced current and induced voltage will form the discharge area at the corner and tip of the structure, resulting in the discharge damage [7]. Furthermore, the accidents such as the breakdown of the lightning current through the insulation plate and cable rack will take place. The greater efforts shall be made to improve such method.

3 ) When introducing downwards from the top of the structure, the wire clamp or steel belt shall be used to bind OPGW with concrete (steel) poles by segments [8]. By such method, the grounding of OPGW at multiple points are achieved; however, the contact place is liable to oxidation and corrosion as the time goes by, resulting in the gas discharge damage and with the prejudice to the measurement of the resistance of the ground grid of the substation.

4) Overhead introduction of OPGW downwards with $10 \mathrm{kV}$ composite insulator post. At present, the rubber insulation pad is often used for overhead insulation in China. Such rubber insulation pad is $20 \mathrm{~mm}$ thick, resulting in the uneasy installation and fixation. Due to the in-service aging, it shall be replaced on a regular basis. The method that OPGW downwards with $10 \mathrm{kV}$ composite insulator post is applied. The insulator post is used to fix connection cable connector box and over cable racks of OPGW so that there is no non-mounting fixing point between the OPGW and any metal member, so as to eliminate the potential safety hazard in the discharge (See Figure 2). In most cases, the insulator post shall be installed at the interval of $1.5 \mathrm{~m}$. This method can solve the existing problem, but increase the number of support will bring the substation maintenance more difficult.

5 ) The OPGW is connected to the ordinary optical cable on the terminal tower, and the ordinary optical cable is directly buried and introduced into the substation [9]. By such method, the substation structure is separated so as to avoid the over-current damage to the OPGW; however, the lightning strike is still possible in case of the long distance from the terminal tower to the substation; furthermore, the directly buried avoid the risk of the damage by the external force.

\section{Grounding measures of OPGW in longmen framework}

OPGW grounding on the existing substation structure, which was divided into the upper ground part and lower ground part, grounding connectors and aluminum using mergers and acquisitions at the top line, to realize the connection of OPGW strain clamp nearby and top grounding plate of structure

[10]. The lower grounding part is often exists near OPGW excess cable rack by combine the drainage wire clamp and aluminum alloy wire to realized the connection of OPGW with structure bottom part and substation grounding plate. The connection part have the hidden heating danger on the connection part due to the constantly rusting or loose joint about the connection part. 
For the OPGW grounding problem, we had carried out research and offer the solution to reinforced the OPGW grounding on the top part of the substation structure after, please see the details as follows:

1) At the front end of the strain clamp,reinforcing rod strain clamp is used to connect with OPGW cable and avoid these hidden danger on the segment clearance while introducing downwards to the structure by using the advantage of great holding force and long segment length about reinforcing rod strain clamp.

2) The type of aluminum cable used on lower grounding part is bigger than OPGW cable, and connect with grounding power net by using introducing downwards insulated protection pipe.The application of insulation pipe can solve the hidden danger of discharge damage in the electric discharging area forming by the induced current and induced current in the edges and tip of structure.

3 ) Connect with the grounding wire by using aluminum terminal clamp and double nuts fixing to achieve reliable connection of grounding grip.

4) Insulation structure can be used to support OPGW junction box and OPGW excess cable rack to avoid grounding. The adoption of this method eliminate the hidden gap electric discharge caused by thunder-lighting and short circuit, which solve the years confusing problem and realized the expected subject of the paper.

\section{Conclusion}

As the OPGW cable is different from ordinary cable, it is required to achieve the separation of wire and signal after the introduction of the substation structure; at the same time, the grounding for lightning is also required because of the frequent occurrence of the burnout of the optical cable due to the electric discharge as a result of the problems in the grounding device or construction.

In combination with the practical conditions of the project, after analysis and study in the construction site, the solution "reinforcing the connection with OPGW cable by using armored rod strain clamp and lead insulation pipe down to the substation structure to realized the reliable connection with grounding power net" is brought up and solve the practical problem, which solve the years confusing gap discharge problem and hidden danger.

\section{References}

[1] Fang Yuesheng,Zeng Fanxing,Development trend of technological application in OPGW,J.Study on Optical Communications, vol. 6(2014),p.48-51

[2] LI Peng,Cui Jian,Fu Jie,Analysis of asymmetric short-circuit current distribution in OPGW overhead transmission line system,J. Journal of North China Electric Power University,vol. 4(2014),p.68-70

[3] Fan Qiang, Zhang Zhemin,Chen Wenzhang et al, Research on Lightning Detection and Location in Power Grid Based on OPGW,J.Advances of Power System \& Hydroelectric Engineering, vol. 11(2014),p.69-71

[4] Jiang Haiming,Zhu Shenghua,Lu Yuan et al,Research and Implementation on Standardization of OPGW Access Substation Architecture,J.Telecommunications for Electric Power System, vol. 1(2013),p.26-28

[5] Cui Hong,Xia Chengjun,Du Tiancang et al,Numerical Analysis of OPGW Damage by Lightning and Structural Optimization,J. High Voltage Apparatus,vol. 6(2010),p.54-59

[6] Liu Hongjun.,Research and discussion on lightning protection and grounding technology in OPGW optical cable in electric power system,J.Digital Technology and Application,vol. 12(2011),p.35-37 
[7] Cai Ling,Zhou Zhou,Research and discussion on introduction of OPGW optical cable below gantry of substation,J. Hunan Electric Power, vol. 3(2012),p.43-45

[8] Peng Xiangyang,Liu Yigang,Zhang Huiqiang,On-site Measurement Based Analysis and Simulation of Abnormal Heating of Optical Fiber Composite Overhead Ground Wires,J. Power System Technology, vol. 2(2012),p.206-211

[9] Li Xinhua,Discussion on grounding of OPGW optical cable introduced into substation,J.Science and Technology Information, vol. 25(2013),p.391-392

[10] Yang Ziqiang, Liu Jiannong,Suggestions on improvement of erection method for prevention of lightning strike against OPGW,J. Telecommunications for Electric Power System,vol. 2(2011),p.61-63 